Research Article

\title{
Sensor Network Disposition Facing the Task of Multisensor Cross Cueing
}

\author{
Ce Pang, Shucai Huang, Yan Zhao, Daozhi Wei, and Jinchang Liu
}

Air and Missile Defense College, Air Force Engineering University, Xian 710051, China

Correspondence should be addressed to Ce Pang; 18392447996@163.com

Received 2 December 2016; Accepted 18 May 2017; Published 28 June 2017

Academic Editor: Federica Caselli

Copyright (C) 2017 Ce Pang et al. This is an open access article distributed under the Creative Commons Attribution License, which permits unrestricted use, distribution, and reproduction in any medium, provided the original work is properly cited.

In order to build the sensor network facing the task of multisensor crossing cueing, the requirements of initiating cueing and being cued are analyzed. Probability theory is used when building models, then probability of sensor cueing in the case of target moving is given, and, after that, the best distance between two sensors is calculated. The operational environment is described by normal distribution function. In the process of distributing sensor network, their elements, operational environment demand of cueing, and the probability of sensor network coverage are considered; then the optimization algorithm of sensor network based on hypothesis testing theory is made. The simulation result indicates that the algorithm can make sensor network which is required. On the basis of that, the two cases, including targets that make linear motion and orbit motion, are used to test the performance of the sensor network, which show that the sensor network can make uninterrupted detection on targets through multisensor cross cuing.

\section{Introduction}

Multisensor cross cueing technology is put forward and developed to solve the problem of dynamic management of sensors, meaning that when sensors carry out tasks together, they can cue with each other, and the cued sensors can get the information about targets directly to get further information about targets [1-3]. Multisensor cross cueing can greatly improve the detecting ability of sensors in the intelligence monitoring and scouting system, and it plays a role mainly in two aspects: on one hand, when sensors break down or they cannot detect targets any more, sensors can cue other sensors to hand over target-detecting tasks, by which targets can be detected successively [4]; on the other hand, sensor can cue other sensors to detect targets together so that the detection accuracy can be improved or extra information can be gotten [5].

Multisensor cross cueing technology is regarded as the important development trend in the sensor field up to 2015 in America [6]. After making the definition, the function, and the method of multisensor cross cueing $[4,7,8]$ clear, the technology enters the application phase. McCarthy Panel [9] uses multisensor cross cueing technology to simulate and verify the scene where SAR and MTI detect the whole target area together. Katsilieris and Narykov make study on the functions of cueing between different kinds of sensors and put forward the sensor-managing methods in the condition of cueing $[10,11]$. Salvagnini and Muratore discuss sensor cueing opportunity and methods when there are different kinds of sensors in multiple platforms [12, 13]. Pang et al. make a study on the problem of how the multisensor cross cueing technology is applied on target tracking [14].

It is known that there is no introduction on the problem of sensor network deployment facing multisensor cross cueing in the former study, but multisensor cross cueing takes place in the sensor network, requiring good connection of the sensor network to satisfy the need of communication. Whether the performance of the sensor network is well or not can influence the effect of multisensor cross cueing.

In this paper, sensors are radars, and the sensor network is built facing multisensor cross cueing. Models are made based on probability theory, and the probability of multisensor cross cueing is defined. The best distance between two sensors is calculated, and the optimization algorithm based on hypothesis test to optimize the sensor network is put forward. In the simulation, the process of building the sensor 


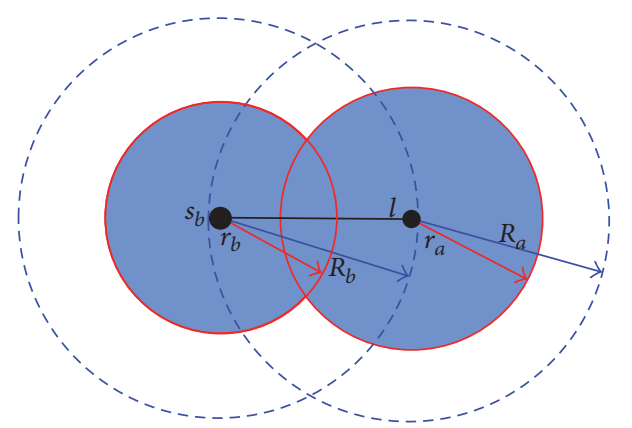

Figure 1: The relationship of two sensors when effective cueing happens.

network and the process of multisensor cross cueing are shown.

The probability of multisensor cross cueing models is built based on probability theory, with the best distance between two sensors calculated. The combat environment is described using normal distribution function. After this work, the optimization algorithm based on hypothesis test to optimize the sensor network is put forward. The simulation indicates that the method the paper introduces can get the sensor network to satisfy the need of multisensor cross cueing. What is more, the process of multisensor cross cueing is shown, which indicates that the multisensor cross cueing technology can adapt to the combat reality, making targets detected successively and having a good application prospect.

\section{Basic Theory of Sensor Cueing}

2.1. Inspiring Conditions of Cueing between Two Sensors. Assume that the distance between sensor $s_{a}$ and sensor $s_{b}$ is $l$, the detecting radius and the communicating radius of $s_{a}$ are $r_{a}$ and $R_{a}$, and the detecting radius and the communicating radius of $s_{b}$ are $r_{b}$ and $R_{b}$. The sufficient condition of cross cueing between $s_{a}$ and $s_{b}$ is

$$
\begin{aligned}
& R_{a} \geq l, \\
& R_{b} \geq l .
\end{aligned}
$$

In this paper, the cueing way between two sensors is "beam cueing," and the effective cueing is defined as follows: if cross cueing can happen between two sensors and the cued sensor can catch the target, the cueing is effective cueing, or the cueing is invalid cueing. The condition of effective cueing is

$$
r_{a}+r_{b} \geq l
$$

The happening condition schematic diagram of cross cueing between $s_{a}$ and $s_{b}$ is as Figure 1 describes.

\subsection{The Cueing Model between Sensors Based on Probability} Theory. Assume that $s_{a}$ is the cueing sensor and $s_{b}$ is the cued sensor. In order to simplify the model, in this paper, $r=r_{a}=$ $R_{a}=r_{b}=R_{b}$. Assume that when the target is in the area of $S_{0}=S_{a} \cap S_{b}$ and " $A$ " stands for the event that $s_{a}$ cues $s_{b}$, in

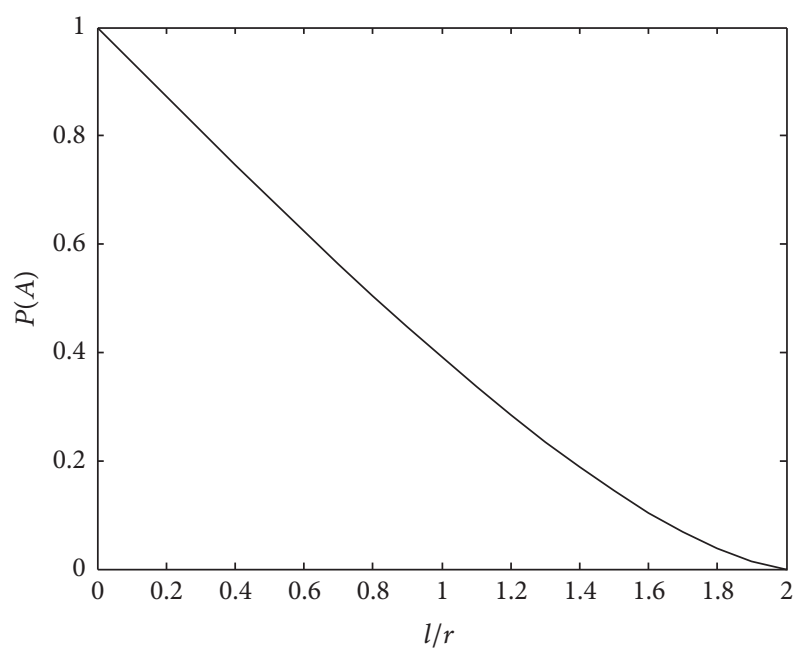

FIgure 2: The Curve of Function $P(A)$.

terms of probability theory, then probability of occurrence of $A$ is as follows:

$$
P(A)=\frac{S_{0}}{S_{a}} .
$$

In the formula, $S_{a}$ stands for the detecting area of $s_{a}$ and $S_{b}$ stands for the detecting area of $s_{b}$.

After geometric calculation, we can get

$$
P(A)=\frac{2 r^{2} \operatorname{arcos}(l / 2 r)-l \sqrt{r^{2}-l^{2} / 4}}{\pi r^{2}} .
$$

Regard $l / r$ as $x$ coordinate and $P(A)$ as $y$ coordinate, and then the relationship of $P(A)$ and $l / r$ is as shown in Figure 2.

2.3. The Multisensor Cross Cueing Model When Targets Move. For the reason that targets move fast when coming, this paper pays attention to the problem of multisensor cross cueing when targets move fast. If a target is flying away from the detecting area of $s_{a}, s_{a}$ needs to cue other sensors to hand over the task of detecting this target. If $s_{b}$ is determined to accept the task, after $s_{b}$ start to detect the target, $s_{a}$ will not detect the target any more.

Firstly, the cueing time is defined as follows.

Assume that at the time of $T$, the distance of one target and $s_{a}$ is $d_{a}(T)$, and the distance of this target and $s_{b}$ is $d_{b}(T)$. Assume that, at the time of $T+1$, the distance of one target and $s_{a}$ is $d_{a}(T+1)$, and the distance of this target and $s_{b}$ is $d_{b}(T+1)$. When $s_{a}$ is detecting the target, if $d_{a}(T+1)>d_{a}(T)$ and $d_{a}(T)>0.7 r, s_{a}$ will produce the need of cueing, and then it will cue other sensors to take over the detecting task. When $d_{b}(T+1)<d_{b}(t)$ and the target is in the area of $S_{0}=S_{a} \cap S_{b}$, $s_{b}$ has the ability to take over the detecting task, and it can be cued by $s_{a}$.

Assume that " $B$ " sands for the event that $s_{b}$ catch the target successfully. As $B$ is carried out after $A$, in terms of probability theory, the probability of $B$ is as follows:

$$
P(B)=P(A) *\left(1-\frac{\left(S_{a} \cap S_{b}\right)}{S_{b}}\right) .
$$


For $S_{a}=S_{b}$, we can get

$$
P(B)=P(A)(1-P(A)) .
$$

Regard $l / r$ as $x$ coordinate and $P(B)$ as $y$ coordinate, and then the relationship of $P(B)$ and $l / r$ is as shown in Figure 3.

In Figure 3, we can get $\max (P(B))=0.25$ and $P(A)=0.5$ and $l=0.81 r$ when $P(B)=0.25$.
When $s_{a}$ needs to cue other sensors, if there are several sensors which can be cued, $s_{a}$ chose the cued sensor based on the probability $p(B)$.

In addition, the detecting radius is usually smaller than the communicating radius [15]; that is to say, $r_{a}<R_{a}$ and $r_{b}<R_{b}$.

What is more, ignore the assumption $r=r_{a}=R_{a}=r_{b}=$ $R_{b}$, and then there is

$$
P(A)=\frac{\left[r_{a}^{2} \operatorname{arcos}\left(\left(l^{2}+r_{a}^{2}-r_{b}^{2}\right) / 2 l r_{a}\right)+r_{b}^{2} \operatorname{arcos}\left(\left(l^{2}+r_{b}^{2}-r_{a}^{2}\right) / 2 l r_{b}\right)-r_{a} r_{b} \sqrt{1-\left(\left(r_{a}^{2}+r_{b}^{2}-l^{2}\right) / 2 r_{a} r_{b}\right)^{2}}\right]}{\pi r_{a}^{2}} .
$$

It can be seen that $p(A)$ and $p(B)$ are three-dimensional functions of $r_{a}, r_{b}$, and $l$, and when $l=100$, the functions are as Figures 4 and 5 describe.

\section{Build the Sensor Network}

3.1. Combat Environment Scenarios. Assume that, in the combat environment, the coordinate of our position is $(-a, 0)$, the coordinate of the enemy is $(a, 0)$, and there are $n$ sensors which need to be deployed to detect targets of the enemy. The distribution of our position and the position of the enemy is described in Figure 6, and the shadow area is what we should watch to detect targets from the enemy.

Based on the rule that targets should be detected as soon as possible, sensors should be deployed near the enemy, but sensors are so near the enemy that they can be destroyed by the weapons form the enemy. According to the rule of " $3 \sigma$ " [16], assume that, in the rectangular area $D$, the strategic value of each position obeys the regular as follows:

$$
\begin{aligned}
& f(x, y) \\
& \quad=\frac{1}{2 \pi(a / 3)(b / 3)} \exp \left[-\frac{x^{2}}{2(a / 3)^{2}}-\frac{y^{2}}{2(b / 3)^{2}}\right] .
\end{aligned}
$$

3.2. The Building Rules of Sensor Network. Building the sensor network, which is the basis for multisensor cross cueing, makes a direct impact on the performance of multisensor cross cueing. In order to satisfy the need of multisensor cross cueing, when building the sensor network, the total number of degrees [17] in the sensor network should remain pretty large, making good connectivity between every sensor.

When deploying sensors in the area $D$, rules should be obeyed as follows:

(1) Sensors tend to be deployed where there is high strategic value.

(2) In order to satisfy the need of multisensor cross cueing, the distance of each two sensors tends to be $l=0.81 r$.

(3) The coverage of the sensor network should remain large.
Rule one is designed to meet the combat environment in this paper; rule two is designed to satisfy the need of multisensor cross cueing; rule three is designed due to the rule in the previous papers.

\subsection{The Steps of Building Sensor Network}

To Build the Earning Degree Function. Assume $p_{1}=p(A)$, $p_{2}=p(B), p_{3}=p(C)$, and the position of $s_{i}$ is $\left(x_{i}, y_{i}\right)$. There are $k_{i}$ sensors which can communicate directly with $s_{i}$, and the set is $c_{i}=\left\{s_{i 1}, s_{i 2}, \ldots, s_{i k_{i}}\right\}$, in which $s_{i j}$ is the number $j$. The probability of cross cueing between $s_{i}$ and $s_{i j}$ is $p_{2}\left(s_{i j}\right)$. Assume that, in the position $\left(x_{i}, y_{i}\right)$, the strategic value is $f\left(x_{i}, y_{i}\right)$, and then the earning degree of $s_{i}$ can be calculated as follows:

$$
\begin{aligned}
g\left(s_{i}\right)= & \omega_{1} f\left(x_{i}, y_{i}\right)+\omega_{2} \sum_{j=1}^{k_{i}}\left(p_{2}\left(s_{i j}\right)\right) \\
& +\left(1-\omega_{1}-\omega_{2}\right) \frac{S}{4 a b} .
\end{aligned}
$$

In the function, $\omega_{1}, \omega_{2} \in[0,1]$, which is the weight, and $S$ is the area which the sensor network covers.

In this paper, $\omega_{1} f\left(x_{i}, y_{i}\right)$ is called strategic earning, $\omega_{2} \sum_{j=1}^{k_{i}}\left(p_{2}\left(s_{i j}\right)\right)$ is called cueing earning, $\left(1-\omega_{1}-\omega_{2}\right)(S / 4 a b)$ is called coverage earning, and $g\left(s_{i s}\right)$ is called the total earning.

To Initialize the Positions of Sensors. At the time of $t=0$, the grid method [17] is used to initialize the positions of sensors, the area is divided into $N \times M$ grids, and, at each vertex, there is one sensor.

To Optimize the Positions of Sensors. Assume, at the time of $t$, the vector, whose direction is from $\left(x_{i}(t), y_{i}(t)\right)$ to $(0,0)$, is $\overrightarrow{e_{i}}$. $s_{i}$ can move through the direction $\overrightarrow{e_{i}}$ or $-\overrightarrow{e_{i}}$, and the speed is $v_{s_{i}}(t)$, which is calculated as follows:

$$
v_{s_{i}}(t)=\frac{f(0,0)-f\left(x_{i}(t), y_{i}(t)\right)}{f(0,0)} v .
$$

In the function, $v$ is a constant value. 


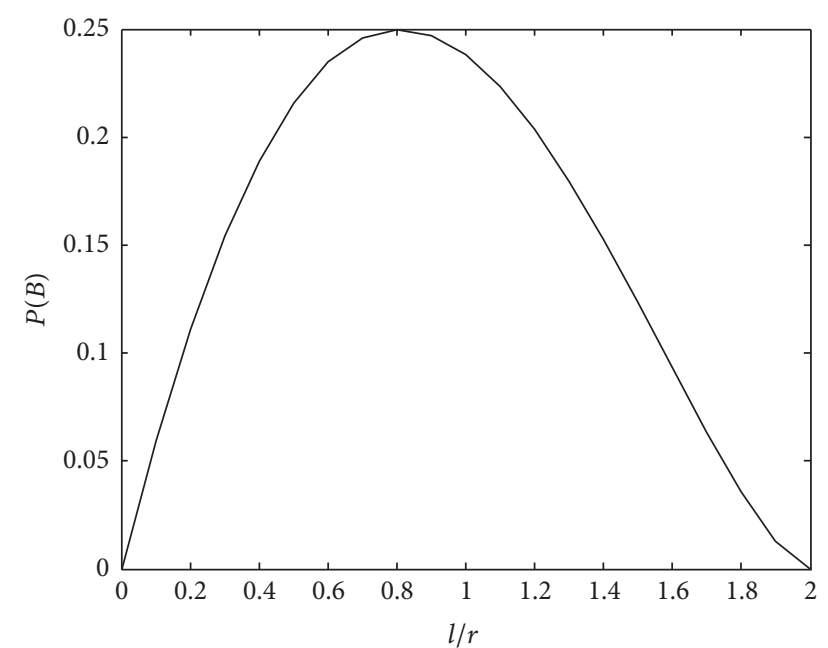

FIgure 3: The Curve of Function $P(B)$.

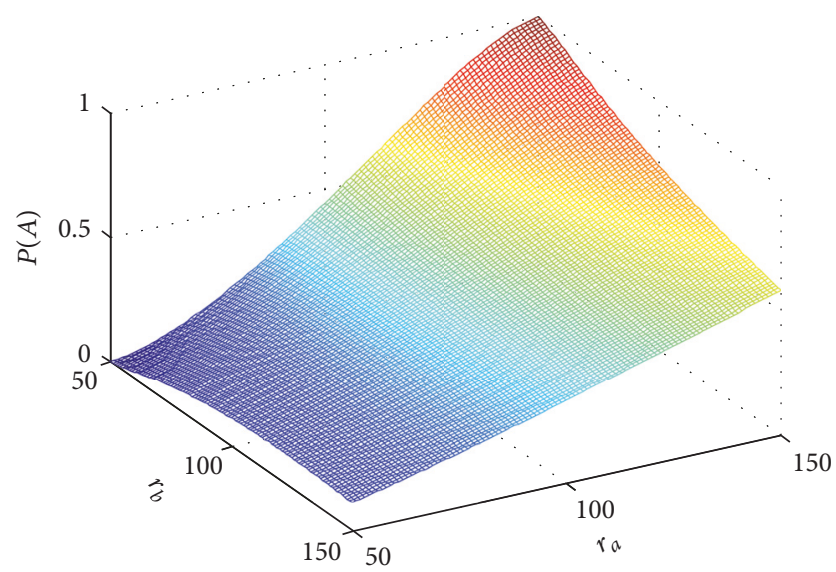

FIgure 4: The Curve of Function $P(A)$.

Move through the direction $\overrightarrow{e_{i}}$, then the strategic earning of $s_{i}$ can increase, and the coverage earning can decrease; move through the direction $-\overrightarrow{e_{i}}$, then the strategic earning of $s_{i}$ can decrease, and the coverage earning can increase (in the case where each two sensors have common detecting area).

According to the statistical detection theory, assume that the case $H_{k}(t)=1$ is representative for the total earning increase if one sensor is moving through the direction $\overrightarrow{e_{i}}$, and the case $H_{k}(t)=0$ is representative for the total earning increase if one sensor is moving through the direction $-\overrightarrow{e_{i}}$. Assume that, at the time $T$, the probability of moving through the direction $\overrightarrow{e_{i}}$ is $q_{i}(t)$, and the probability of the increase of the total earning is $o_{i}(t)\left(0.5<o_{i}(t) \leq 1\right)$; assume that the probability of moving through the direction $-\overrightarrow{e_{i}}$ is $1-q_{i}(t)$ and the probability of the increase of the total earning is $1-o_{i}(t)$. After moving, the total earning of $s_{i}$ is $f\left(x_{i}(t+1), y_{i}(t+1)\right)$.

(1) When $f\left(x_{i}(t+1), y_{i}(t+1)\right) \geq f\left(x_{i}(t), y_{i}(t)\right)$, update functions as follows.

At the time of $t$, the probability of the increase of the total earning after moving is

$$
u_{i}(t)=q_{i}(t) * o_{i}(t)+\left(1-q_{i}(t)\right) *\left(1-o_{i}(t)\right)
$$

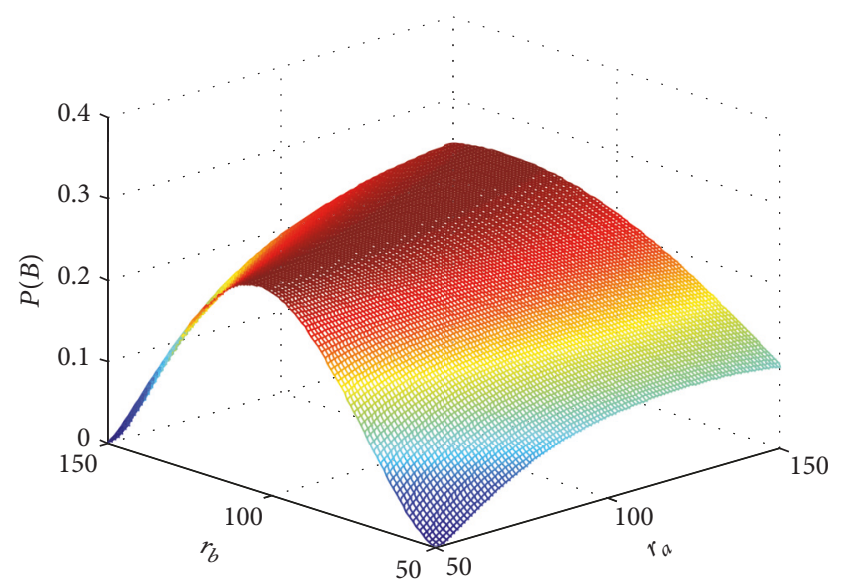

Figure 5: The Curve of Function $P(B)$.

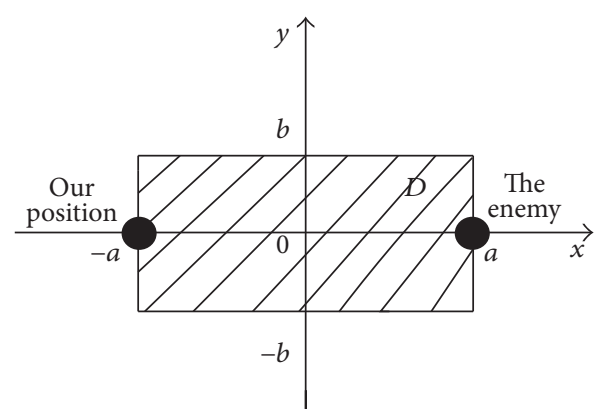

FIgURE 6: The position relationship of the enemy and our side.

At the time of $t+1$,

$$
\begin{aligned}
& q_{i}(t+1)=q_{i}(t) * \frac{o_{i}(t)}{u_{i}(t)} \\
& o_{i}(t+1)=o_{i}(t) .
\end{aligned}
$$

The following is to prove $q_{i}(t)<q_{i}(t+1)<1$.

(1)

$$
\begin{aligned}
& q_{i}(t+1)>q_{i}(t) \Longleftarrow \\
& \frac{o_{i}(t)}{q_{i}(t) * o_{i}(t)+\left(1-q_{i}(t)\right) *\left(1-o_{i}(t)\right)}>1 \Longleftarrow \\
& 2 q_{i}(t)+\frac{1}{o_{i}(t)}-\frac{q_{i}(t)}{o_{i}(t)}-1<0 \Longleftarrow \\
& \left(2+\frac{1}{o_{i}(t)}\right)\left(q_{i}(t)-1\right)<0 .
\end{aligned}
$$

(2) The function $q_{i}(t+1)<1$ is obviously right.

That is to say, if the move at the time of $t$ can prove that $H_{k}(t)=1$ is right, then, at the time of $t+1, q_{i}(t+1)$ will increase, and $o_{i}(t+1)$ will be the lager number.

(2) When $f\left(x_{i}(t+1), y_{i}(t+1)\right)<f\left(x_{i}(t), y_{i}(t)\right)$, update functions as follows.

At the time of $t$, the probability of the increase of the total earning after moving is

$$
u_{j}^{-}(t)=\left(1-q_{i}(t)\right) * o_{i}(t)+q_{i}(t) *\left(1-o_{i}(t)\right) .
$$


At the time of $t+1$,

$$
\begin{aligned}
& q_{i}(t+1)=q_{i}(t) * \frac{\left(1-o_{i}(t)\right)}{u_{j}^{-}(t)} \\
& o_{i}(t+1)=1-o_{i}(t) .
\end{aligned}
$$

The following is to prove $0<q_{i}(t+1)<q_{i}(t)$. (1)

$$
\begin{gathered}
q_{i}(t+1)<q_{i}(t) \Longleftarrow \\
\frac{q_{i}(t) *\left(1-o_{i}(t)\right)}{\left(1-q_{i}(t)\right) * o_{i}(t)+q_{i}(t) *\left(1-o_{i}(t)\right)}<q_{i}(t) \Longleftarrow \\
\frac{1-o_{i}(t)}{\left(1-q_{i}(t)\right) * o_{i}(t)+q_{i}(t) *\left(1-o_{i}(t)\right)}<1 \Longleftarrow \\
\left(1-q_{i}(t)\right)\left(1-2 o_{i}(t)\right)<0 \\
\because\left(0.5<o_{i}(t) \leq 1\right)
\end{gathered}
$$

$\therefore$ The function is right.

(2) The function $q_{i}(t+1)>0$ is obviously right.

That is to say, if the move at the time of $t$ cannot prove that $H_{k}(t)=1$ is right, then, at the time of $t+1, q_{i}(t+1)$ will decrease, and $o_{i}(t+1)$ will be the smaller number.

To Build the Sensor Network. At the time of $t=t_{\text {end }}$, positions of sensors will not change any more and then connect sensors which can communicate with each other to make edges and calculate the weight of each edge according to function (4) and function (9), and, finally, a chart $G(V, E, W)$ can be made, which is the sensor network.

\section{The Simulation and the Analysis}

In the process of simulation, parameters are as follows: $a=$ $50, b=15, M=7, N=3, r=8, q(0)=0.6, o(0)=0.6$, $v=5$, and $T=50$. In the beginning, sensors are deployed according to grid method [18], and the distribution is as Figure 7 describes. The red circles are sensors, and if there is a line between two sensors, it means cross cueing can happen between the two sensors.

In the simulation, the value of $\omega_{1}, \omega_{2}$ can exert direct influence on the optimized result of the sensor network. If $w_{1}=1$ and $w_{2}=0$, the most optimal result is when all sensors are at the position of $(0,0)$; if $w_{1}=0$ and $w_{2}=1$, the most optimal result is when each distance of two sensors is $0.81 r$; if $w_{1}=w_{2}=0$, the most optimal result is when there is no common area between each two sensors.

At the time of $t$, the sum of degrees of all vertexes is $k_{0}=$ 64 , and the average distance of all vertexes is $l_{0}=11.29$.

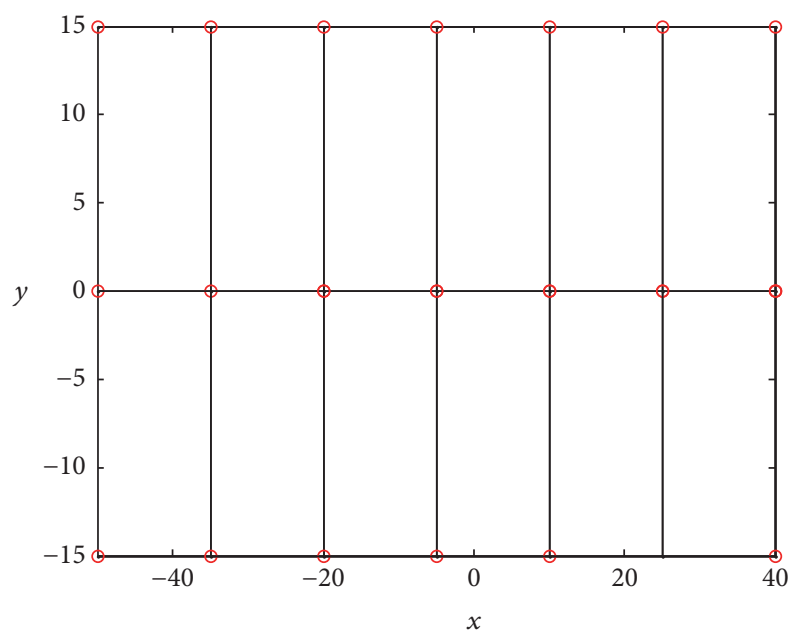

Figure 7: Positions of sensors at the beginning of iteration.

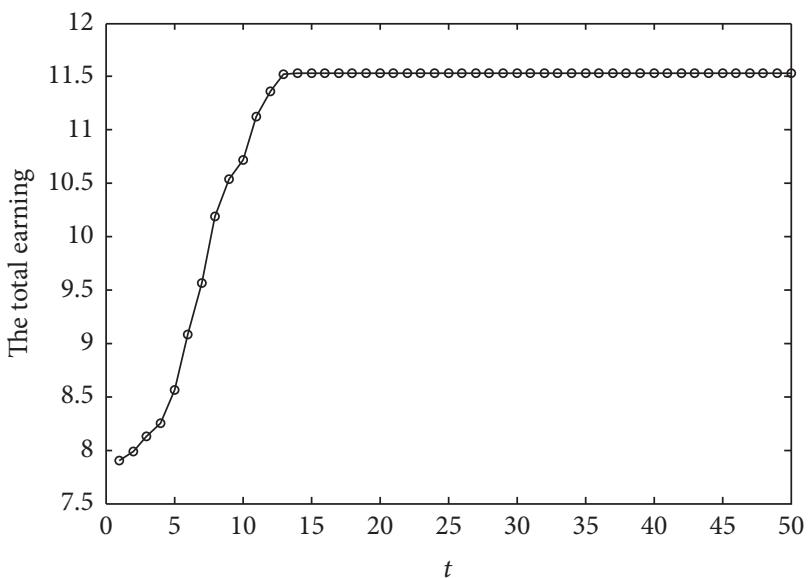

FIgURE 8: The iteration process of the optimization using the method in this paper.

\subsection{The Optimization of the Sensor Network}

The Optimization of the Sensor Network When $w_{1}=0.99997$, $w_{2}=0.00003$, and $w_{3}=0$. In this case, the coverage earning is ignored, and the iteration process is as Figure 8 describes.

The optimal result is as Figure 9 describes.

In Figure 9, the sum of degrees of all vertexes is $k_{1}=431$, and the average distance of all vertexes is $l_{1}=5.76$. It can be known that when sensor stops moving, the distance of two sensors is near $l=0.81 r=0.648$.

From Figure 9, it can be known that when ignoring the coverage earning, all sensors tend to move to the position of $(0,0)$. The detecting area of the sensor network will decrease, and the reason is mainly as follows: the first one is the highest strategic earning at the position of $(0,0)$ attracts every sensor to move to itself; the other one is that sensors move close to try to get more cueing earning. Although all sensors tend to move to the position of $(0,0)$, there is some distance between each 
two sensors, and the reason is as follows: each sensor tries to adjust the distance with others to $0.81 r$ under rule two.

The Optimization of the Sensor Network When $w_{1}=0.99994$, $w_{2}=0.00003$, and $w_{3}=0.00003$. In this case, the coverage earning is considered, and the iteration process is as Figure 10 describes.

The optimal result is as Figure 11 describes.

In Figure 11, the sum of degrees of all vertexes is $k_{2}=124$, and the average distance of all vertexes is $l_{2}=9.38$.
Compared to Figure 9, the sensor network can get lager coverage area in Figure 11, it can satisfy the need of multisensor cross cueing, and there are a number of sensors at the position of $(0,0)$ at the same time, which correspond to the combat reality.

In this paper, multisensor cross cueing is analyzed based on the sensor network of Figure 11. In Figure 11, coordinates of all sensors is as Table 1 describes, and the weight of each edge is as function (17) describes.

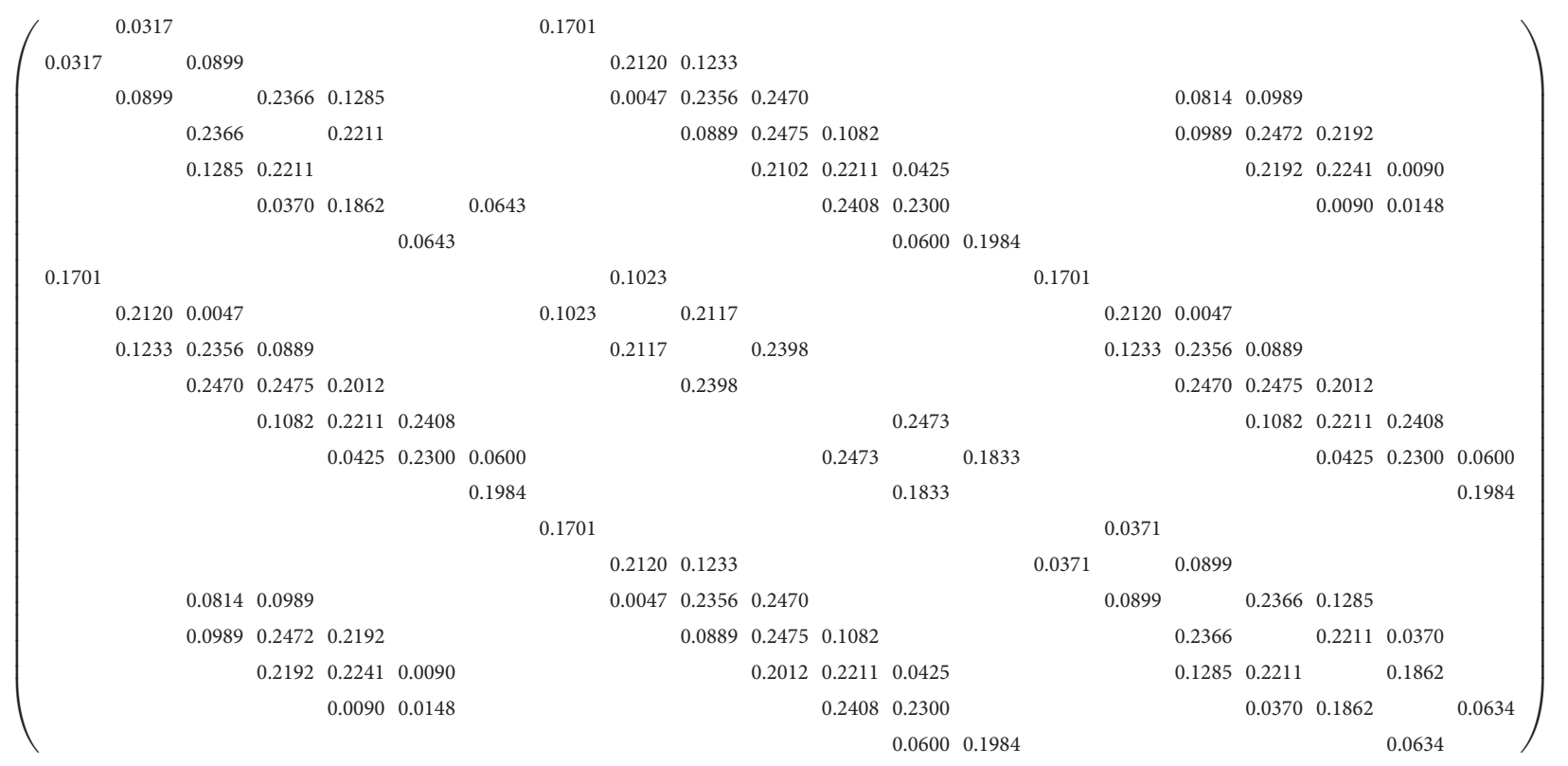

\subsection{The Process of Multisensor Cross Cueing}

The Process of Multisensor Cross Cueing at the Case When the Target Makes Linear Motion. Assume that the target is a UCAV, at the time of $T=0$, the target takes off from the position of the enemy $(50,0)$, and the speed is $V=0.5 / \mathrm{s}$. The target moves to $(-10,0)$ through the direction of $-x$, and then it moves back to $(50,0)$ through $x$. At the time of $T$, if $0 \leq$ $T \leq 120$, the coordinate of the target is $(50-V * T, 0)$, and if $120 \leq T \leq 240$, the coordinate of the target is $(-60+V * T, 0)$.

The process of multisensor cross cueing is as Figure 12 describes. The horizontal coordinate is the time $T$, and the vertical coordinate is the sensor which is responsible for the detection to the target.

The Process of Multisensor Cross Cueing at the Case When the Target Makes Sinusoidal Motion. Assume that the target is a UCAV and, at the time of $T=0$, the target takes off from the position of the enemy $(50,0)$, making sinusoidal motion. The flight path is $y=10 \sin (0.04 x+\pi)$, and, at the time of $T=0$, the coordinate of the target is $(50-t, 10 \cos (0.04(50-t)+$ $\pi))$.

The process of multisensor cross cueing is as Figure 13 describes. The horizontal coordinate is the time $T$, and the vertical coordinate is the sensor which is responsible for the detection to the target.
The two simulations can illustrate that the sensor network can make multisensor cross cueing smooth and successive detection to the target.

\section{Conclusion}

In this paper, the probability of multisensor cross cueing model is built based on probability theory, with the best distance between two sensors calculated. The combat environment is described using normal distribution function. After this work, the optimization algorithm based on hypothesis test to optimize the sensor network is put forward. The simulation indicates that the method the paper introduces can get the sensor network to satisfy the need of multisensor cross cueing. What is more, the process of multisensor cross cueing is shown, which indicates that the multisensor cross cueing technology can adapt to the combat reality, making targets detected successively and having a good application prospect.

\section{Conflicts of Interest}

The authors declare that there are no conflicts of interest regarding the publication of this paper. 


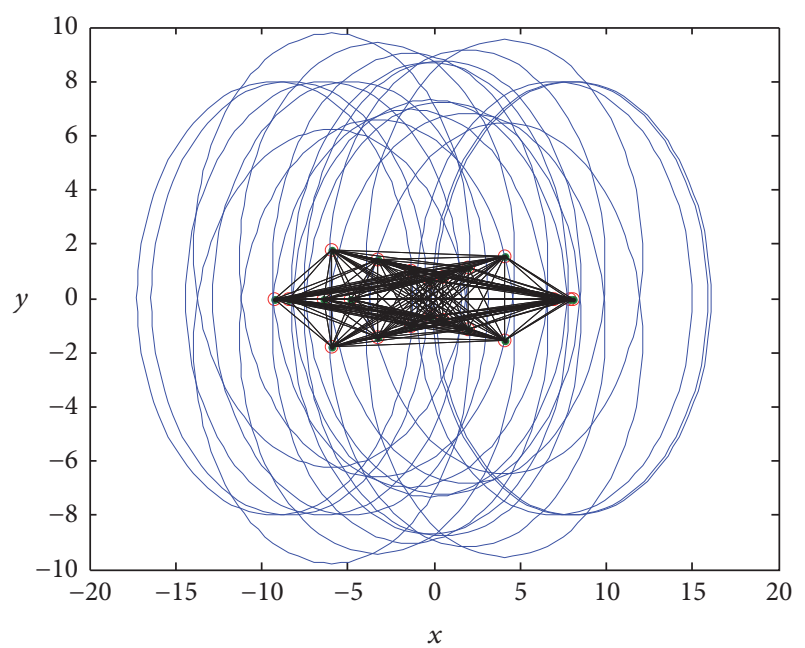

FIGURE 9: The optimized result of sensor network.

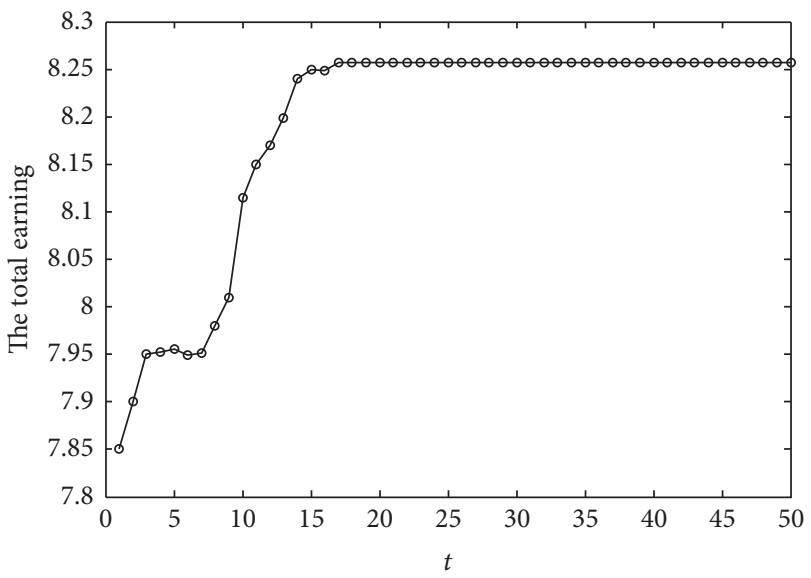

FIGURE 10: The iteration process of the optimization using the method in this paper.

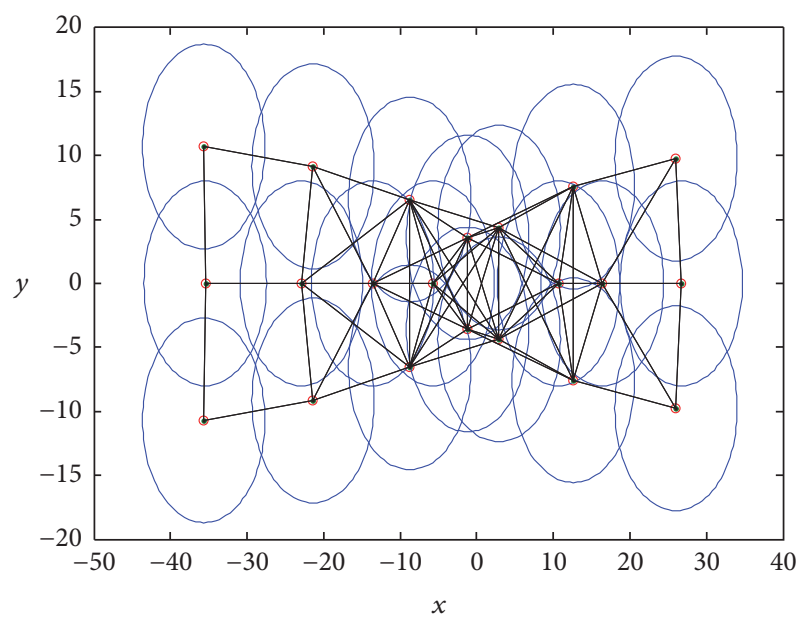

FIGURE 11: The optimized result of sensor network.

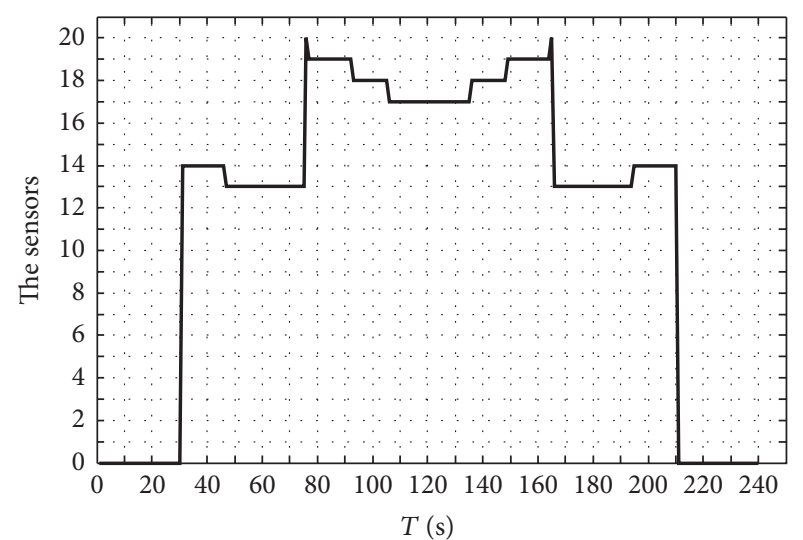

FIGURE 12: Procession of multisensor cross cueing when the UCAV is coming.

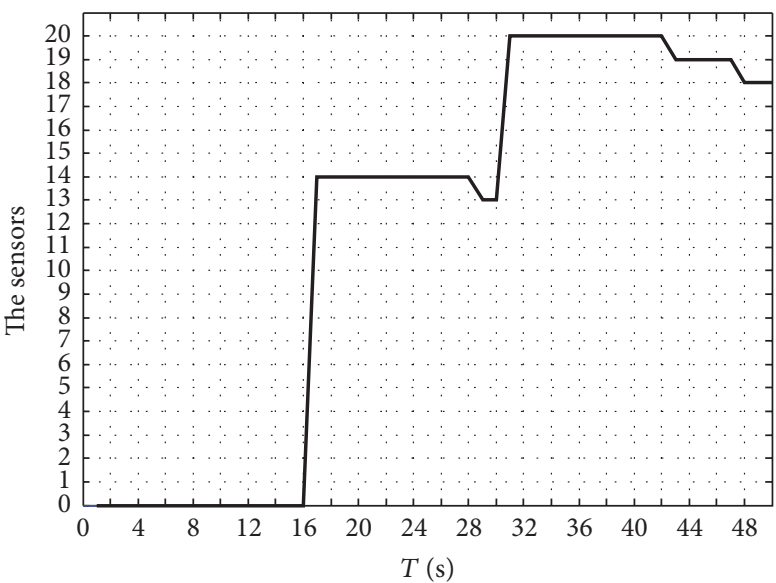

FIGURE 13: Procession of multisensor cross cueing when the UCAV is coming.

TABLE 1: Coordinates of sensors.

\begin{tabular}{lc}
\hline Sensor & Coordinate \\
\hline 1 & $(-35.65,10.67)$ \\
2 & $(-21.37,9.16)$ \\
3 & $(-8.74,6.56)$ \\
4 & $(-1.20,3.60)$ \\
5 & $(2.92,4.38)$ \\
6 & $(12.61,7.57)$ \\
7 & $(26.03,9.76)$ \\
8 & $(-35.46,0)$ \\
9 & $(-22.89,0)$ \\
10 & $(-13.60,0)$ \\
11 & $(-5.72,0)$ \\
12 & $(10.67,0)$ \\
13 & $(16.43,0)$ \\
14 & $(26.72,0)$ \\
15 & $(-35.65,-10.67)$ \\
16 & $(-21.37,-9.16)$ \\
17 & $(-8.74,-6.56)$ \\
18 & $(-1.20,3.60)$ \\
19 & $(2.92,-4.38)$ \\
20 & $(12.61,-7.57)$ \\
21 & $(26.03,-9.76)$ \\
\hline
\end{tabular}




\section{Acknowledgments}

This work was supported by National Natural Science Fund of China under Grant no. 61573374.

\section{References}

[1] G. W. Ng, K. H. Ng, and L. T. Wong, "Sensor management - control and cue," in Proceedings of the 3rd International Conference on Information Fusion, FUSION 2000, Paris, France, 2000.

[2] Y. He, X. Guan, and G. Wang, "Survey on the progress and prospect of multisensor information fusion," Journal of Astronautics, vol. 26, no. 4, pp. 524-530, 2005.

[3] H. Fan, Z. Long, S. Huang, Y. Li, and M. Gao, "Development and study of multi-sensor cross cueing technology," Aerodynamic Missile Journal, vol. 2, pp. 79-84, 2012.

[4] M. Avalle, "Studies and simulations on sensor fusion and cueing for fighter application," in Advisory Group for Aerospace Research and Development, North Atlantic Treaty Organization, pp. 224-229, Cananda Communication Group, Cananda, 1996, Section IV.

[5] G. C. Crystal, "An Approach to Realizing the Potential of Information Operations," IEEE Transactions on BAE, vol. 3, pp. 33-40, 2001.

[6] H. Zhaochao and S. Huan, "Analysis of multi-sensors detecting and tracking $\mathrm{cm}$ based on near-space," Aero Weaponry, vol. 2, pp. 3-7, 2010.

[7] T. Peli, M. Young, R. Knox et al., "Feature level sensor fusion," XDDARPA, Atlantic Aerospace Electronics Corporation, 1999.

[8] D. Strǒmberg, "A sensor-independent sensor-oriented tracking architecture," in Proceedings of the IEEE Information Decision and Control Conference, pp. 111-116, Adelaide, SA, Australia, 1999.

[9] L. Bush, "Semi-automated cueing of predator UAV operators from radar moving target (MTI) data," MIT Lincoln Laboratory, Intelligence Surveillance Reconnaissance (ISR) Systems Group, Integrated Sensing and Decision Support (ISDS) Laboratory, Jan. 24, 2006.

[10] F. Katsilieris, "Sensor management for surveillance and tracking: an operation perspective," Delft: Delft University of Technology, 2015.

[11] A. S. Narykov, O. A. Krasnov, and A. Yarovoy, "Effectivenessbased radar resource management for target tracking," in Proceedings of the International Radar Conference, IEEE, Lille, France, October 2014.

[12] P. Salvagnini, F. Pernici, M. Cristani et al., "Non-myopic information theoretic sensor management of a single pantiltzoom camera for multiple object detection and tracking," Computer Vision and Image Understanding, vol. 134, pp. 74-88, 2015.

[13] M. Muratore, R. T. Silvestrini, and T. H. Chung, "Simulation analysis of UAV and ground teams for surveillance and interdiction," Journal of Defense Modeling and Simulation: Applications, Methodology, Technology, vol. 11, no. 2, pp. 125-135, 2014.

[14] C. Pang, S. Huang, and J. Liu, "Multi-sensor cross cueing technology and its application in target tracking," Chinese Journal of Astronautics, vol. 38, no. 4, pp. 401-409, 2017.

[15] R. A. Hooshmand and S. Soltani, "Fuzzy optimal phase balancing of radial and meshed distribution networks using BF-PSO algorithm," IEEE Transactions on Power Systems, vol. 27, no. 1, pp. $47-57,2012$.
[16] Z. Lu, M. Li, and X. Ji, "Research on radar searching volume based on mutil-sensor cooperation technology," Aeronautical Computing Technique, vol. 16, no. 3, pp. 30-35, 2015.

[17] J. Lin, "Network analysis of China's aviation system, statistical and spatial structure," Journal of Transport Geography, vol. 22, pp. 109-117, 2012.

[18] Y. Zou and K. Chakrabarty, "Sensor deployment and target localization in distributed sensor networks," ACM Transactions on Embedded Computing Systems, vol. 3, no. 1, pp. 61-91, 2004. 


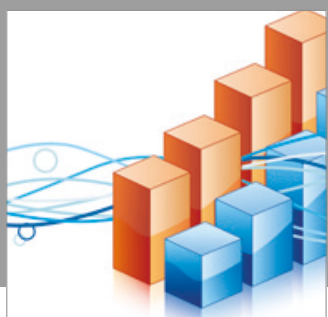

Advances in

Operations Research

vatersals

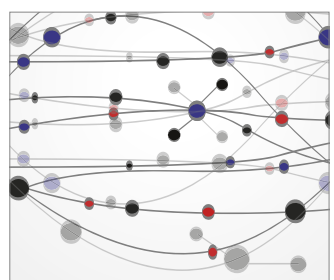

\section{The Scientific} World Journal
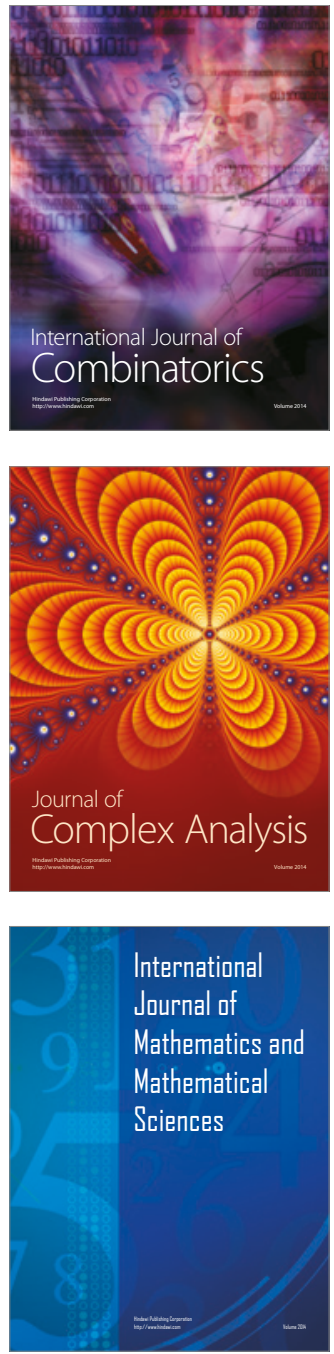
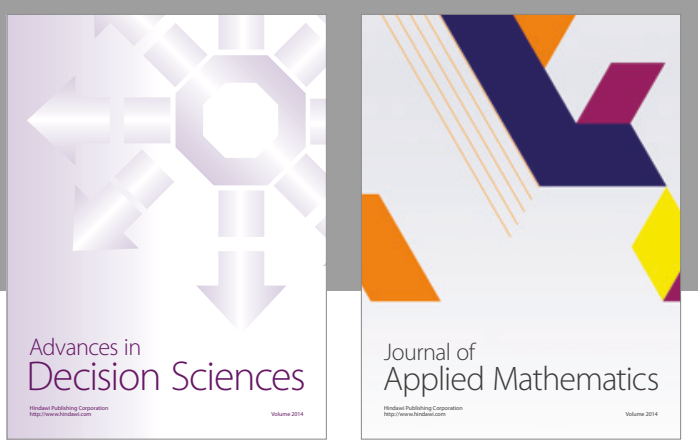

Algebra

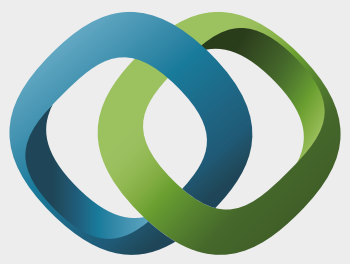

\section{Hindawi}

Submit your manuscripts at

https://www.hindawi.com
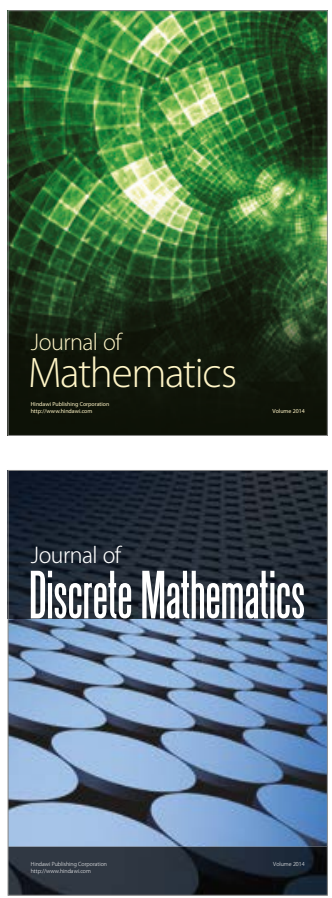

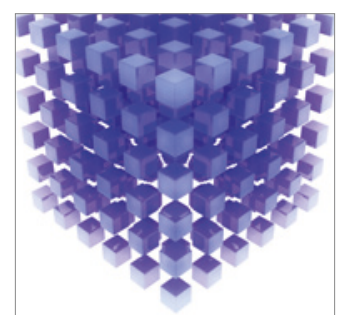

Mathematical Problems in Engineering
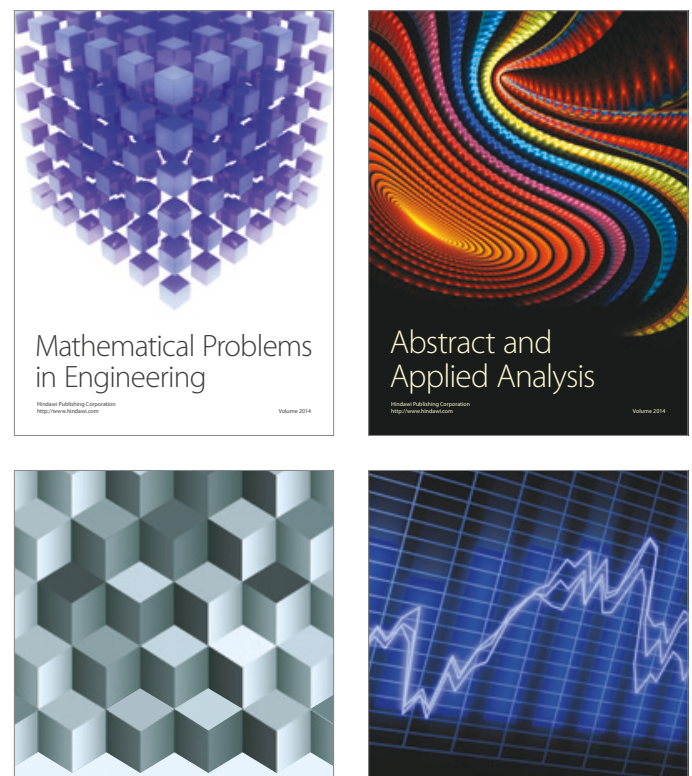

Journal of

Function Spaces

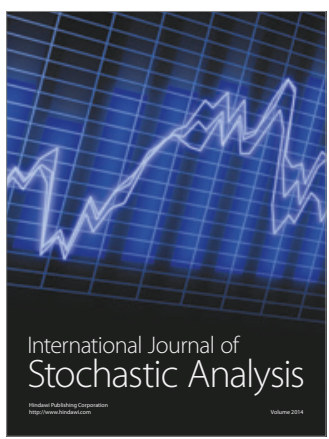

Probability and Statistics
